\title{
Correction to: Learning nonlinear input-output maps with dissipative quantum systems
}

\author{
Jiayin Chen ${ }^{1} \cdot$ Hendra I. Nurdin ${ }^{1}$
}

Received: 7 July 2019 / Accepted: 10 September 2019 / Published online: 10 October 2019

(c) Springer Science+Business Media, LLC, part of Springer Nature 2019

\section{Correction to: Quantum Information Processing (2019) 18(7):198 https://doi.org/10.1007/s11128-019-2311-9}

The original version of this article unfortunately contained errors in the proofs of Lemma 1, Lemma 5 and Proposition 1. Corrections to the proofs of Lemmas 1 and 5 and Proposition 1 are given below:

\section{Correction to [1, Lemma 5]}

In the proof of Lemma 5, it was incorrectly claimed that $\left.\left(T^{(1)} \otimes T^{(2)}\right)\right|_{H_{0}\left(\mathbb{C}^{2^{n}}\right)}=$ $\left.\left.T^{(1)}\right|_{H_{0}\left(\mathbb{C}^{2^{n}}\right)} \otimes T^{(2)}\right|_{H_{0}\left(\mathbb{C}^{2^{n}}\right)}$. However, since the constituting subsystems are taken to be non-interacting and initialized in a product state [1, $\$$, paragraph 1], this erroneous argument in the proof is unnecessary. A correct and simpler argument showing that $T^{(1)} \otimes T^{(2)}$ is again convergent when restricted to product states of the subsystems, and the polynomial algebra $\mathscr{F}$ consists of fading memory maps, is the following. To show the convergence property when restricted to product states of the subsystems, given any two initial product states $\rho_{1,0} \otimes \rho_{2,0}$ and $\sigma_{1,0} \otimes \sigma_{2,0}$, we have

$$
\begin{aligned}
& \left\|\rho_{1, k} \otimes \rho_{2, k}-\sigma_{1, k} \otimes \sigma_{2, k}\right\|_{2} \\
& \leq\left\|\coprod_{j=1}^{k}\left(T^{(1)}\left(u_{j}\right) \otimes T^{(2)}\left(u_{j}\right)\right) \rho_{1,0} \otimes\left(\rho_{2,0}-\sigma_{2,0}\right)\right\|_{2} \\
& +\left\|\prod_{j=1}^{k}\left(T^{(1)}\left(u_{j}\right) \otimes T^{(2)}\left(u_{j}\right)\right)\left(\rho_{1,0}-\sigma_{1,0}\right) \otimes \sigma_{2,0}\right\|_{2}
\end{aligned}
$$

The original article can be found online at https://doi.org/10.1007/s11128-019-2311-9.

$凶$ Hendra I. Nurdin

h.nurdin@unsw.edu.au

1 School of Electrical Engineering and Telecommunications, The University of New South Wales (UNSW), Sydney, NSW 2052, Australia 


$$
\begin{aligned}
= & \left\|\rho_{1, k}\right\|_{2}\left\|\left(\prod_{j=1}^{k} T^{(2)}\left(u_{j}\right)\right)\left(\rho_{2,0}-\sigma_{2,0}\right)\right\|_{2} \\
& +\left\|\sigma_{2, k}\right\|_{2}\left\|\left(\coprod_{j=1}^{k} T^{(1)}\left(u_{j}\right)\right)\left(\rho_{1,0}-\sigma_{1,0}\right)\right\|_{2} \\
\leq & 2\left(1-\varepsilon_{2}\right)^{k}\left\|\rho_{1, k}\right\|_{2}+2\left(1-\varepsilon_{1}\right)^{k}\left\|\sigma_{2, k}\right\|_{2} \leq 2\left(1-\varepsilon_{2}\right)^{k}+2\left(1-\varepsilon_{1}\right)^{k},
\end{aligned}
$$

where the last two inequalities follow from the property that for any density operator $\rho$, $\|\rho\|_{2} \leq 1$. Furthermore, the subsystems are initialized in a product state $\rho_{-\infty}^{(1)} \otimes \rho_{-\infty}^{(2)}$. Therefore, the terms in the output functionals $F^{T^{(1)}}+\lambda F^{T^{(2)}}$ and $F^{T^{(1)}} F^{T^{(2)}}$ are products of quantum expectations of the form $\operatorname{Tr}\left(Z^{\left(j_{1}\right)}\left(\vec{\prod}_{k=0}^{\infty} T^{(1)}\left(u_{-k}\right)\right) \rho_{-\infty}^{(1)}\right)$ for $j_{1}=1, \ldots, n_{1}$ or $\operatorname{Tr}\left(Z^{\left(j_{2}\right)}\left(\vec{\prod}_{k=0}^{\infty} T^{(2)}\left(u_{-k}\right)\right) \rho_{-\infty}^{(2)}\right)$ for $j_{2}=1, \ldots, n_{2}$. Since $T^{(1)}$ and $T^{(2)}$ satisfy the conditions in Lemma 3 , these quantum expectations are continuous with respect to $\|\cdot\|_{w}$. The fading memory property follows from the fact that finite sums and products of continuous elements are again continuous.

\section{Correction to [1, Proposition 1]}

In the argument showing that $T_{K}(x)$ satisfies the conditions in Lemma 3 for all $x \in$ $[0,1], \tilde{T}$ was incorrectly claimed to be a CPTP map. However, the proof only requires $\tilde{T}$ to be bounded. This is automatically satisfied since $\tilde{T}$ is a linear operator on a finite dimensional normed space.

\section{Weaker condition for [1, proof of Lemma 1]}

The original proof of Lemma 1 requires the conditions of Theorem 1 to hold. However, the authors noticed that Lemma 1 still holds under the weaker requirement of the convergence property defined in Definition 1 . This implies that a convergent CPTP map induces a unique filter. To see this, for any $\rho \in \mathscr{D}\left(\mathbb{C}^{2^{n}}\right)$ and $j \leq m$,

$$
\begin{aligned}
\left\|S_{j}-S_{m}\right\|_{2} & =\left\|T\left(u_{k}\right) T\left(u_{k-1}\right) \cdots T\left(u_{k-j}\right)\left(\rho-T\left(u_{k-j-1}\right) \cdots T\left(u_{k-m}\right) \rho\right)\right\|_{2} \\
& =\left\|T\left(u_{k}\right) T\left(u_{k-1}\right) \cdots T\left(u_{k-j}\right)\left(\rho-\rho^{\prime}\right)\right\|_{2} .
\end{aligned}
$$

By the convergence property, for any $\varepsilon>0$, there exists $N(\varepsilon) \in \mathbb{N}$ such that for all $j, m \geq N,\left\|S_{j}-S_{m}\right\|_{2}<\varepsilon$. Therefore, $S_{j}$ is Cauchy and $\lim _{j \rightarrow \infty} S_{j}$ exists due to the completeness of $\left(\mathscr{D}\left(\mathbb{C}^{2^{n}}\right),\|\cdot\|_{2}\right)$. It also follows from this argument that $\lim _{j \rightarrow \infty} S_{j}$ is independent of the initial choice of $\rho \in \mathscr{D}\left(\mathbb{C}^{2^{n}}\right)$.

\section{References}

1. Chen, J., Nurdin, H.I.: Learning nonlinear input-output maps with dissipative quantum systems. Quantum Inf. Process. 18(7), 198 (2019) 
Publisher's Note Springer Nature remains neutral with regard to jurisdictional claims in published maps and institutional affiliations. 